\title{
A TM-Pass/TE-Stop Polarizer Based on a Surface Plasmon Resonance
}

\author{
Yuu Wakabayashi, Junji Yamauchi, and Hisamatsu Nakano \\ Faculty of Engineering, Hosei University, 3-7-2 Kajino-cho, Koganei, Tokyo 184-8584, Japan \\ Correspondence should be addressed to Yuu Wakabayashi, yuu.wakabayashi.27@gs-eng.hosei.ac.jp
}

Received 15 June 2010; Accepted 16 July 2010

Academic Editor: Ana Vukovic

Copyright () 2011 Yuu Wakabayashi et al. This is an open access article distributed under the Creative Commons Attribution License, which permits unrestricted use, distribution, and reproduction in any medium, provided the original work is properly cited.

A TM-pass/TE-stop polarizer consisting of a metal film sandwiched between dielectric gratings is investigated using the finitedifference time-domain method. At normal incidence with respect to the grating plane, a transmissivity of more than $94 \%$ and a reflectivity of more than $98 \%$ are obtained at $\lambda=1.55 \mu \mathrm{m}$ for the TM and TE waves, respectively. The extinction ratio is more than $17 \mathrm{~dB}$ over a wavelength range of $1.50 \mu \mathrm{m}$ to $1.75 \mu \mathrm{m}$. A high extinction ratio is maintained at oblique incidence, although the wavelength range shifts towards longer wavelengths. The TM-pass/TE-stop operation is also achieved with a modified structure, in which a dielectric grating is sandwiched between metal films.

\section{Introduction}

There are a great number of papers devoted to the study of light propagation in periodic structures [1]. One of the important applications of the periodic structures is to construct a polarizer, which is used in optical communications and sensing devices. Recently, high transmission of the transverse magnetic (TM) wave through a thin metal film has been suggested and discussed [2-4]. The transmission is closely related to a surface plasmon (SP) resonance [5]. The $\mathrm{SP}$ resonance is realized using a thin metal film sandwiched between dielectric gratings. We should also note that recent interest has been directed toward plasmon waveguides operating at $\lambda \simeq 1.55 \mu \mathrm{m}$ (optical communication band) $[6,7]$.

In this paper, the SP-based enhanced transmission through a thin metal film is investigated in more detail in the optical communication band. The finite-difference timedomain (FDTD) method is used for the analysis. To obtain a high transmissivity for the TM wave and a high reflectivity for the transverse electric (TE) wave with a subsequent high extinction ratio (ER), we appropriately choose the width and thickness of the dielectric grating. In addition to normal incidence with respect to the grating plane, we consider the case of oblique incidence. It was found that the wavelength range, in which the high ER is observed, shifts towards longer wavelengths.

To alleviate the fabrication difficulty, we also deal with a modified structure, in which a dielectric grating is sandwiched between metal films. The TM-pass/TE-stop operation is achieved at $\lambda=1.55 \mu \mathrm{m}$, although the transmissivity is low compared with that obtained from the original structure.

\section{Configuration and Numerical Method}

Figure 1 illustrates the periodic structure of the polarizer, in which a two-dimensional model is treated. The configuration is similar to that treated in $[2,3]$. We illuminate a uniform plane wave of either the TE or the TM wave from the input side and intend to extract the TE wave as a reflected field at the input side and the TM wave as a transmission field at the output side. Note that from a different aspect, a similar structure was also investigated as a low-loss surface plasmon Bragg grating [8].

The refractive indices of the dielectric materials are taken to be $n_{\mathrm{H}}=3.715$ and $n_{\mathrm{L}}=2.049$. The width and thickness of the dielectric grating are designated as $w$ and $t_{\mathrm{d}}$, respectively. The widths of the low- and high-index dielectric regions 


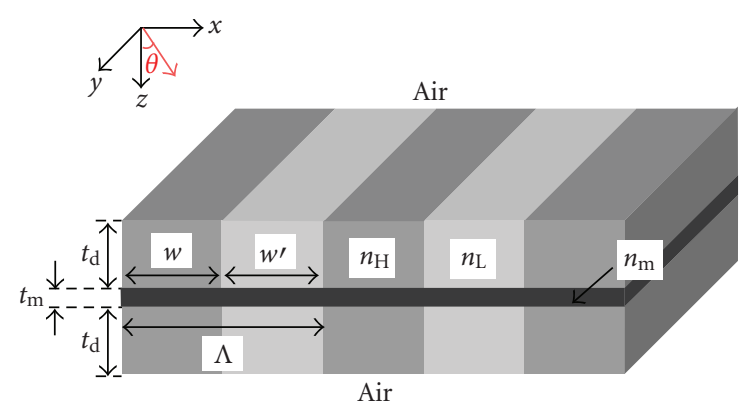

Figure 1: Configuration.

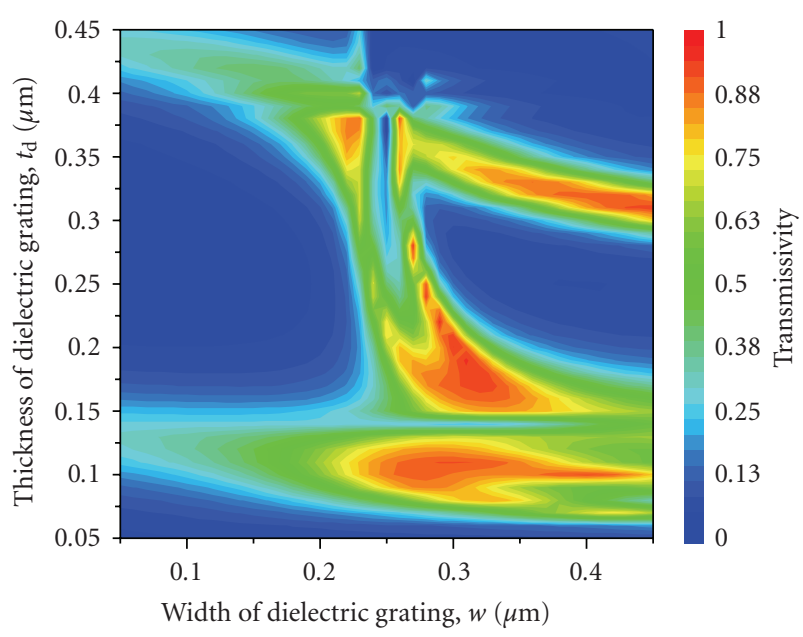

FIgURE 2: Transmissivity of TM wave $(\lambda=1.55 \mu \mathrm{m})$.

are set to be the same, that is, $w=w^{\prime}$. The Drude model is used to express the dispersion of a metal. The refractive index and thickness of the metal (Ag) film are chosen to be $n_{\mathrm{m}}=0.144-j 11.214$ at $\lambda=1.55 \mu \mathrm{m}[9]$ and $t_{\mathrm{m}}=0.03 \mu \mathrm{m}$.

To analyze the present polarizer, we adopt the FDTD method together with the periodic boundary condition, to which the field transformation technique [10] is applied. The piecewise linear recursive convolution technique [11] is employed so as to treat the structure involving the metal film. The numerical parameters are chosen to be $\Delta x=\Delta z=5 \mathrm{~nm}$. We consider the case where a linearly polarized plane wave is incident towards the $+z$ direction whose angle of incidence is defined by $\theta$.

To obtain a high transmissivity for the TM wave with a subsequent high ER, we determine the configuration parameters of dielectric gratings. Figures 2 and 3, respectively, show the transmissivity for the TM wave and the ER at $\lambda=1.55 \mu \mathrm{m}$ as a joint function of $w$ and $t_{\mathrm{d}}$. The angle of incidence is typically fixed to be $\theta=0^{\circ}$. The ER is defined by

$$
\mathrm{ER}=10 \log _{10} \frac{T_{\mathrm{TM}}}{T_{\mathrm{TE}}}
$$

where $T_{\mathrm{TM}}$ and $T_{\mathrm{TE}}$ are the transmissivities for the $\mathrm{TM}$ and TE waves, respectively. Calculation shows that the transmissivity reaches a maximum value of $94 \%$ with an ER of $20 \mathrm{~dB}$

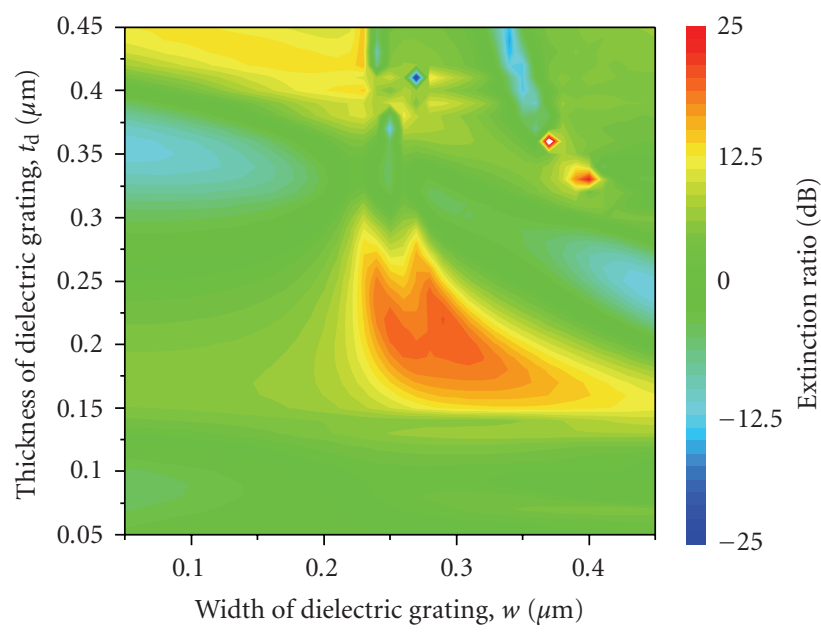

Figure 3: Extinction ratio observed at the output $(\lambda=1.55 \mu \mathrm{m})$.

for $w=0.29 \mu \mathrm{m}$ and $t_{\mathrm{d}}=0.22 \mu \mathrm{m}$. We, therefore, adopt these configuration parameters in the following discussion.

The determined periodicity is consistent with the following relationship at normal incidence, that is, $\theta=0^{\circ}$ :

$$
k_{\mathrm{sp}}=k_{0} \sin \theta \pm \frac{2 \pi}{\Lambda},
$$

where $k_{0}$ is the free-space wavenumber; $\Lambda(=2 w)$ is the periodicity; $k_{\mathrm{sp}}$ is the propagation constant of the SP mode, which can be obtained by the eigenmode analysis. Note that in the eigenmode analysis the refractive index of the dielectric grating is approximately modeled as a homogeneous layer with second-order effective index for the TM wave $\left(n_{\mathrm{TM}}^{(2)}\right)$, which is calculated by the effective medium theory [1]:

$n_{\mathrm{TM}}^{(2)}=\left[n_{\mathrm{TM}}^{2}+\frac{1}{3}\left\{\pi \frac{\Lambda}{\lambda_{0}} f(1-f)\right\}^{2}\left(n_{\mathrm{H}}^{-2}-n_{\mathrm{L}}^{-2}\right)^{2} n_{\mathrm{TM}}^{6} n_{\mathrm{TE}}^{2}\right]^{1 / 2}$,

where $\lambda_{0}$ is the free-space wavelength, and $f$ is the grating fill factor. $n_{\mathrm{TE}}$ and $n_{\mathrm{TM}}$ are the first-order effective indices for the TE and TM waves, which are, respectively, expressed as

$$
\begin{gathered}
n_{\mathrm{TE}}=\left[n_{\mathrm{L}}^{2}+f\left(n_{\mathrm{H}}^{2}-n_{\mathrm{L}}^{2}\right)\right]^{1 / 2}, \\
n_{\mathrm{TM}}=\left[n_{\mathrm{L}}^{-2}+f\left(n_{\mathrm{H}}^{-2}-n_{\mathrm{L}}^{-2}\right)\right]^{-1 / 2} .
\end{gathered}
$$

\section{Wavelength Characteristics}

Figure 4 shows the reflectivity and transmissivity at normal incidence as a function of wavelength. For the TE wave, the reflectivity is close to unity over a wide range of wavelengths. The maximum transmissivity for the TM wave is calculated to be $94 \%$ at $\lambda=1.55 \mu \mathrm{m}$. The ratio of the transmitted field to the reflected field is less than $-30 \mathrm{~dB}$. This means that the nontransmitted power is almost absorbed in the metal film. It should be noted that the double-humped behavior is observed in the transmissivity. This behavior is caused by the fact that the structure supports asymmetric 


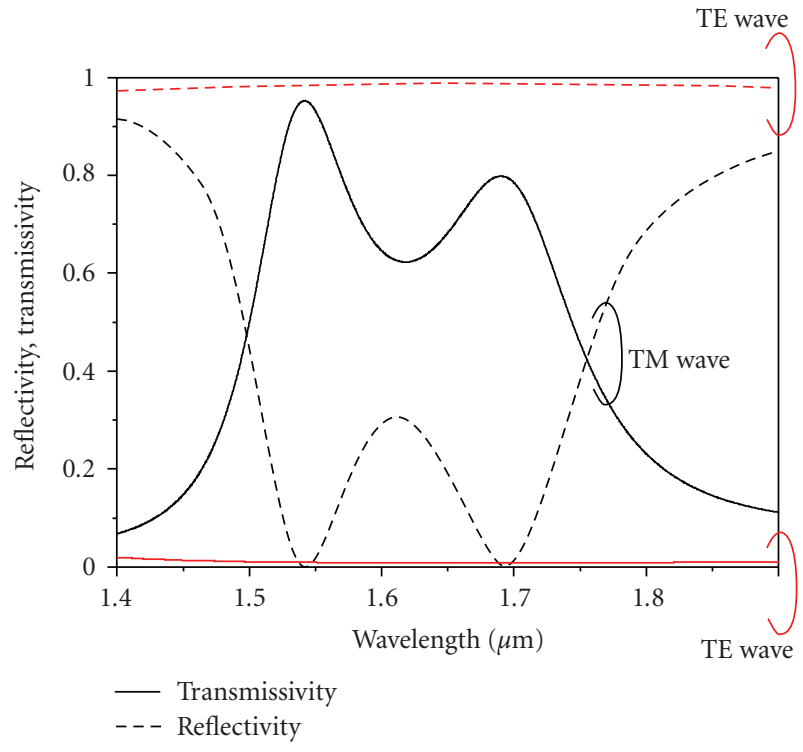

Figure 4: Wavelength characteristics at normal incidence $\left(\theta=0^{\circ}\right)$.

and symmetric modes, which, respectively, correspond to the first and second SP modes [12]. Figures 5(a) and 5(b) show the $H_{y}$-field distributions observed at $\lambda=1.69 \mu \mathrm{m}$ and $1.55 \mu \mathrm{m}$, respectively. Only the fields observed in single cells are presented due to the periodicity. The high-index region is situated from $x=-0.145 \mu \mathrm{m}$ to $x=0.145 \mu \mathrm{m}$. The field in Figure 5(a) exhibits the asymmetric distribution with respect to the middle plane of the metal film, while that in Figure 5(b) exhibits the symmetric distribution.

The ERs observed at the input and output sides are presented in Figure 6. The present polarizer maintains a high ER of more than $17 \mathrm{~dB}$ for the TM wave (at the output) over a wavelength range of $1.50 \mu \mathrm{m}$ to $1.75 \mu \mathrm{m}$. The maximum ER is calculated to be approximately $20 \mathrm{~dB}$ at $\lambda=1.55 \mu \mathrm{m}$. The ER for the TE wave (at the input) is more than $30 \mathrm{~dB}$ at $\lambda=1.55 \mu \mathrm{m}$.

We next study the case of oblique incidence, where $\theta$ is typically set to be $20^{\circ}$. Figures 7 and 8 , respectively, show the transmissivity and the ER each as a function of wavelength. For reference, the data at normal incidence shown in Figures 4 and 6 are again plotted. The maximum transmissivity is obtained at $\lambda=1.62 \mu \mathrm{m}$, and then the triple-humped behavior is observed in this wavelength range. Figure 8 also shows that the region where the high ER is maintained shifts towards longer wavelengths. This behavior can be explained in terms of (2). At normal incidence, only the SP mode whose propagation constant is determined by $\pm 2 \pi / \Lambda$ is excited, propagating in opposite directions along the metaldielectric interface. However, when the angle of incidence is varied from $\theta=0^{\circ}$, this symmetry is broken. In other words, (2) provides the following two relationships:

$$
\begin{aligned}
& k_{\mathrm{sp}}^{+}=k_{0} \sin \theta+\frac{2 \pi}{\Lambda}, \\
& k_{\mathrm{sp}}^{-}=k_{0} \sin \theta-\frac{2 \pi}{\Lambda} .
\end{aligned}
$$

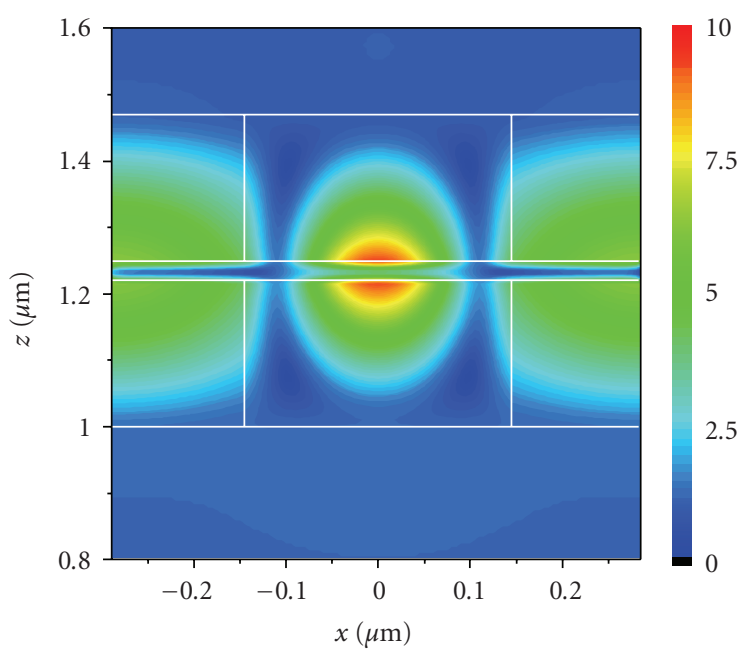

(a)

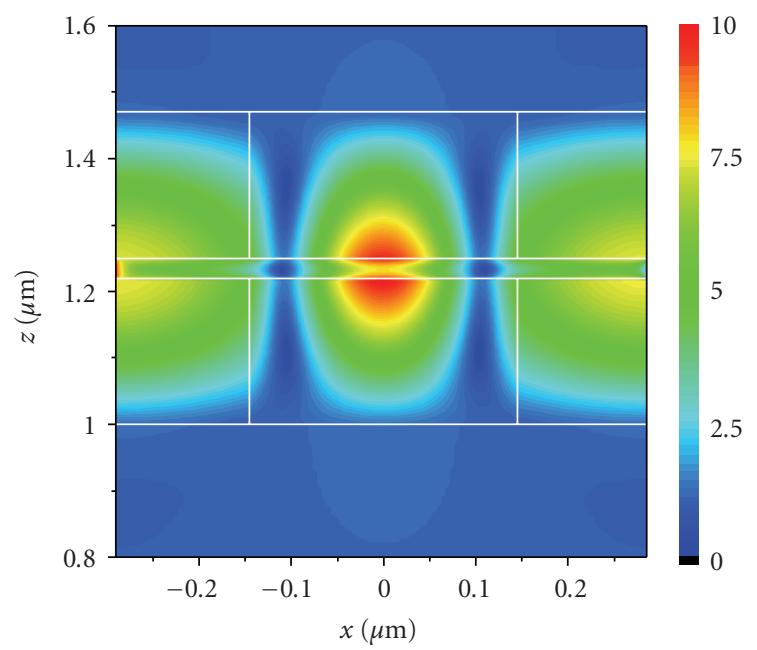

(b)

FIgURE 5: Field distributions at (a) $\lambda=1.69 \mu \mathrm{m}$ and (b) $\lambda=$ $1.55 \mu \mathrm{m}$.

Therefore, the wavelength at which the SP mode is excited at normal incidence is separated into two wavelengths. In Figure 7, the first two modes are excited at $\lambda_{\mathrm{sp}}^{+}=1.53 \mu \mathrm{m}$ and $\lambda_{\mathrm{sp}}^{-}=1.79 \mu \mathrm{m}$, which are separated from $\lambda_{\mathrm{sp}}=1.69 \mu \mathrm{m}$ for $\theta=0^{\circ}$. Only the second mode determined by $k_{\mathrm{sp}}^{-}$can be seen at $\lambda_{\mathrm{sp}}^{-}=1.63 \mu \mathrm{m}$ because the other one $\left(\lambda_{\mathrm{sp}}^{+}=1.33 \mu \mathrm{m}\right)$ is beyond the scale. As a result, the triple-humped behavior of the transmissivity at oblique incidence is observed in this wavelength range.

\section{Modified Structure}

In the preceding section, we have studied the characteristics of the polarizer consisting of the metal film sandwiched between dielectric gratings. This polarizer requires that the structure be symmetric with respect to the metal film to excite the SP mode. It is, however, not easy to fabricate the symmetrical structure at a lightwave frequency, since a set of 


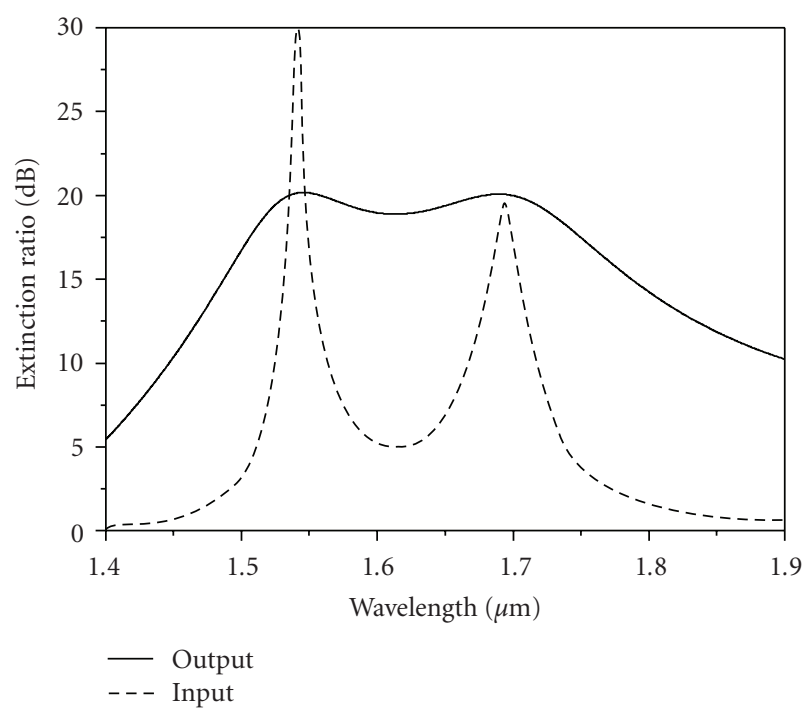

Figure 6: Extinction ratio at normal incidence $\left(\theta=0^{\circ}\right)$.

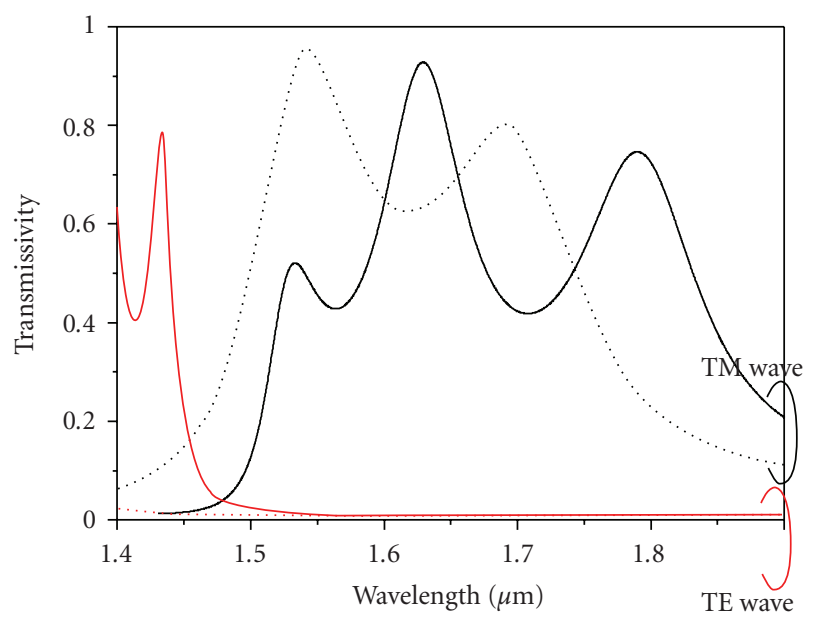

$\begin{array}{ll}\cdots & 0^{\circ} \\ - & 20^{\circ}\end{array}$

FIGURE 7: Transmissivity as a function of wavelength.

dielectric gratings must be placed precisely. In this section, we, therefore, investigate a more practical configuration.

We propose the configuration shown in Figure 9, in which a single dielectric grating is sandwiched between metal films. The refractive indices are the same as those treated in the previous section. It should be noted that the absorption loss is closely related to the thickness of the metal film. Since there exist the two metal films in the modified structure, we have decreased the metal thickness to sufficiently reduce the loss, so that $t_{\mathrm{m}}$ is taken to be $0.01 \mu \mathrm{m}$. To achieve the TM-pass/TE-stop operation at $\lambda=1.55 \mu \mathrm{m}$, we have carried out some preliminary calculations similar to those shown in Figure 2 and finally chose $t_{\mathrm{d}}=0.44 \mu \mathrm{m}$ and $w=w^{\prime}=$ $0.25 \mu \mathrm{m}$.

Figures 10 and 11 show the data corresponding to those shown in Figures 4 and 6, respectively. It was found that a transmissivity of more than $76 \%$ for the TM wave and a

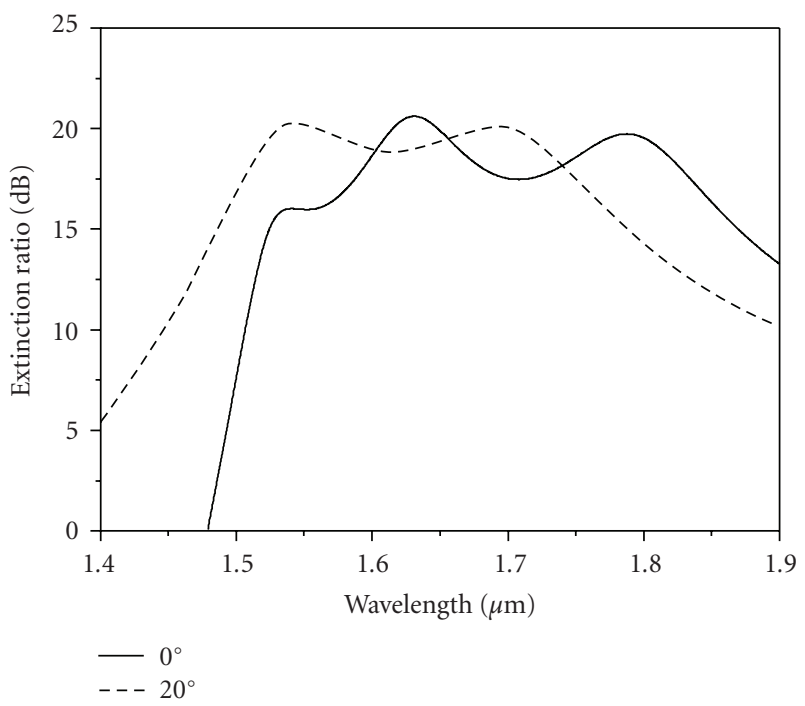

FIgURE 8: Extinction ratio as a function of wavelength.

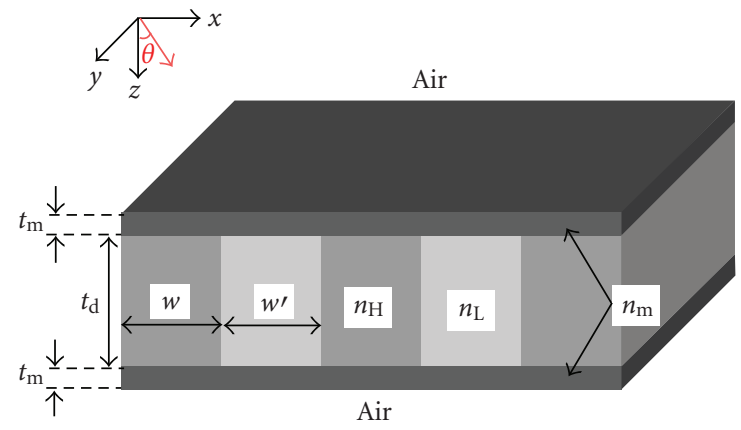

FIgURE 9: Configuration of a modified structure.

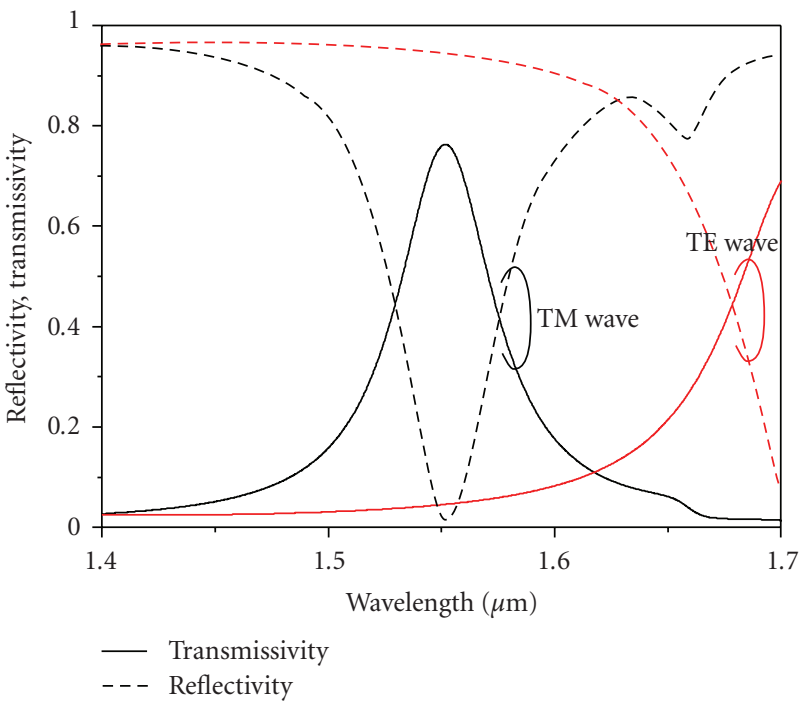

FIGURE 10: Wavelength characteristics. 


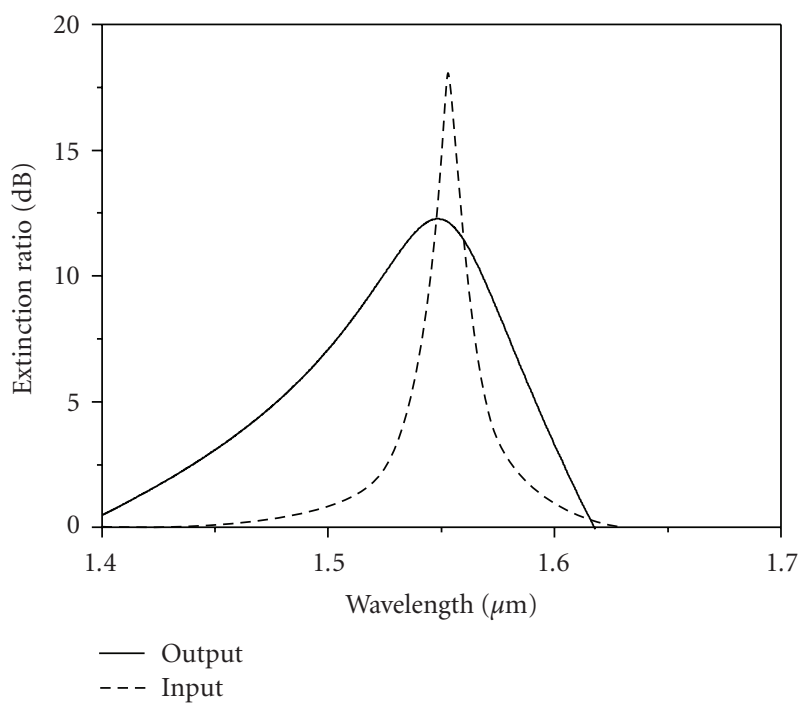

FIGURE 11: Extinction ratio as a function of wavelength.

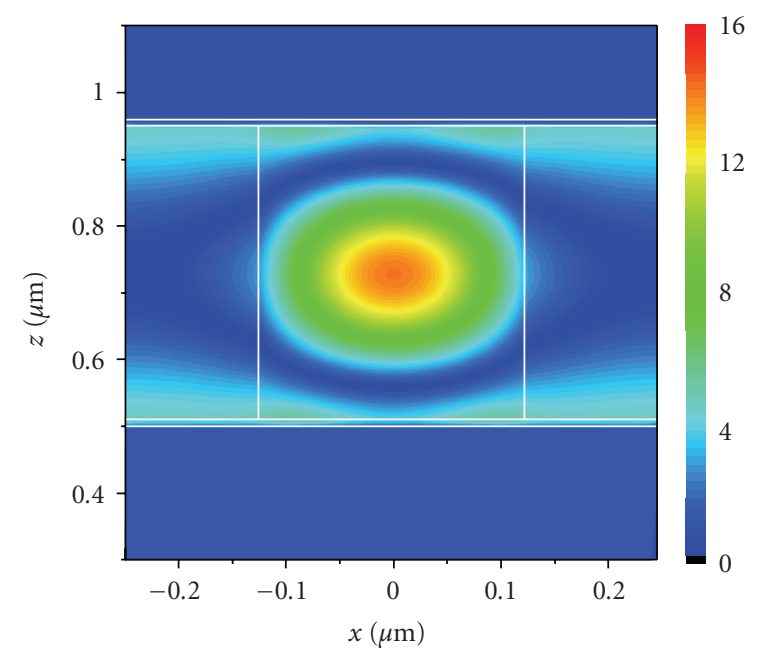

Figure 12: Field distribution $(\lambda=1.55 \mu \mathrm{m})$.

reflectivity of more than $94 \%$ for the TE wave are obtained at $\lambda=1.55 \mu \mathrm{m}$, although the transmissivity is low compared with that obtained from the original structure discussed in Section 3. In contrast with the original structure, the enhanced transmission can be explained in terms of the Fabry-Pérot-like resonance in the cavity between the metal films [13]. The field distribution observed at $\lambda=1.55 \mu \mathrm{m}$ is illustrated in Figure 12, which clearly indicates a standing wave behavior in the high-index region. The ERs at the output and input sides are calculated to be $12 \mathrm{~dB}$ and $18 \mathrm{~dB}$ at $\lambda=1.55 \mu \mathrm{m}$, respectively.

\section{Conclusion}

A TM-pass/TE-stop polarizer using the surface plasmon resonance has been analyzed by the FDTD method. At normal incidence, calculation shows that a transmissivity of more than $94 \%$ for the TM wave is obtained at $\lambda=1.55 \mu \mathrm{m}$. A high ER of more than $17 \mathrm{~dB}$ is observed over a wavelength range of $1.50 \mu \mathrm{m}$ to $1.75 \mu \mathrm{m}$. The high ER region is also maintained at oblique incidence, although the wavelength range shifts towards longer wavelengths.

Further consideration has been devoted to a more practical model in which a dielectric grating is sandwiched between metal films. The TM-pass/TE-stop operation is achieved at $\lambda=1.55 \mu \mathrm{m}$ with a transmissivity of more than $76 \%$. The ER at the output side is calculated to be $12 \mathrm{~dB}$.

\section{Acknowledgments}

The authors would like to thank Mr. Koji Sumida for his basic investigations of the present work. This paper was supported in part by MEXT, Grant-in-Aid for Scientific Research (c) (22560350).

\section{References}

[1] R. Magnusson and D. Shin, "Diffractive optical components," in Encyclopedia of Physical Science and Technology, vol. 4, pp. 421-440, Academic Press, New York, NY, USA, 3rd edition, 2002.

[2] V. M. Fitio and Y. V. Bobitski, "High transmission of system "dielectric grating thin metal film-dielectric grating"," in Proceedings of the 7th International Conference on Laser and Fiber-Optical Networks Modeling (LFNM '05), pp. 163-166, September 2005.

[3] J. Yamauchi, K. Sumida, and H. Nakano, "A TMpass/ TE-stop polarizer consisting of a metal film sandwiched with dielectric gratings," in Proceedings of the 10th International Symposium on Contemporary Photonics Technology, vol. G-15, pp. 93-94, Tokyo, Japan, 2007.

[4] J. Yamauchi, T. Yamazaki, K. Sumida, and H. Nakano, “TM/TE wave splitters using surface plasmon polaritons," in Integrated Photonics and Nanophotonics Research and Applications, Salt Lake City, Utah, USA, July 2007.

[5] J. M. Steele, C. E. Moran, A. Lee, C. M. Aguirre, and N. J. Halas, "Metallodielectric gratings with subwavelength slots: optical properties," Physical Review B, vol. 68, no. 20, Article ID 205103, 7 pages, 2003.

[6] T. Nikolajsen, K. Leosson, and S. I. Bozhevolnyi, "Surface plasmon polariton based modulators and switches operating at telecom wavelengths," Applied Physics Letters, vol. 85, no. 24, pp. 5833-5835, 2004.

[7] K.-Y. Jung, F. L. Teixeira, and R. M. Reano, "Au/SiO 2 nanoring plasmon waveguides at optical communication band," Journal of Lightwave Technology, vol. 25, no. 9, pp. 2757-2765, 2007.

[8] J.-W. Mu and W.-P. Huang, "A low-loss surface plasmonic Bragg grating," Journal of Lightwave Technology, vol. 27, no. 4, pp. 436-439, 2009.

[9] P. B. Johnson and R. W. Christy, "Optical constants of the noble metals," Physical Review B, vol. 6, no. 12, pp. 4370-4379, 1972.

[10] A. Taflove and S. Hagness, Computational Electrodynamics: The Finite-Difference Time-Domain Method, Artech House, Norwood, Mass, USA, 2000.

[11] D. F. Kelley and R. I. Luebbers, "Piecewise linear recursive convolution for dispersive media using FDTD," IEEE Transactions on Antennas and Propagation, vol. 44, no. 6, pp. 792-797, 1996. 
[12] P. Berini, "Plasmon-polariton waves guided by thin lossy metal films of finite width: bound modes of symmetric structures," Physical Review B, vol. 61, no. 15, pp. 10484-10503, 2000.

[13] E. Popov, S. Enoch, G. Tayeb, M. Nevière, B. Gralak, and N. Bonod, "Enhanced transmission due to nonplasmon resonances in one- and two-dimensional gratings," Applied Optics, vol. 43, no. 5, pp. 999-1008, 2004. 

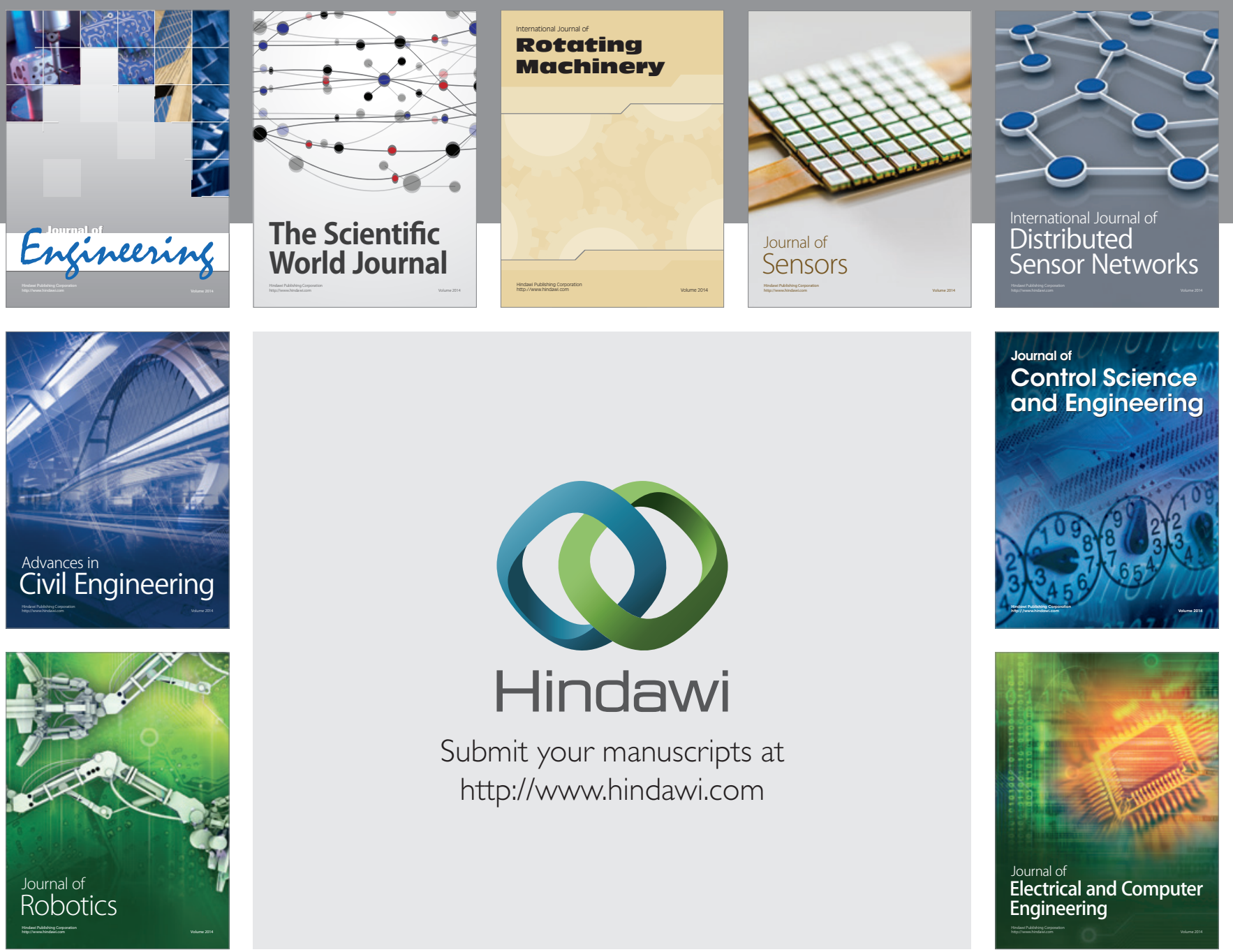

Submit your manuscripts at

http://www.hindawi.com
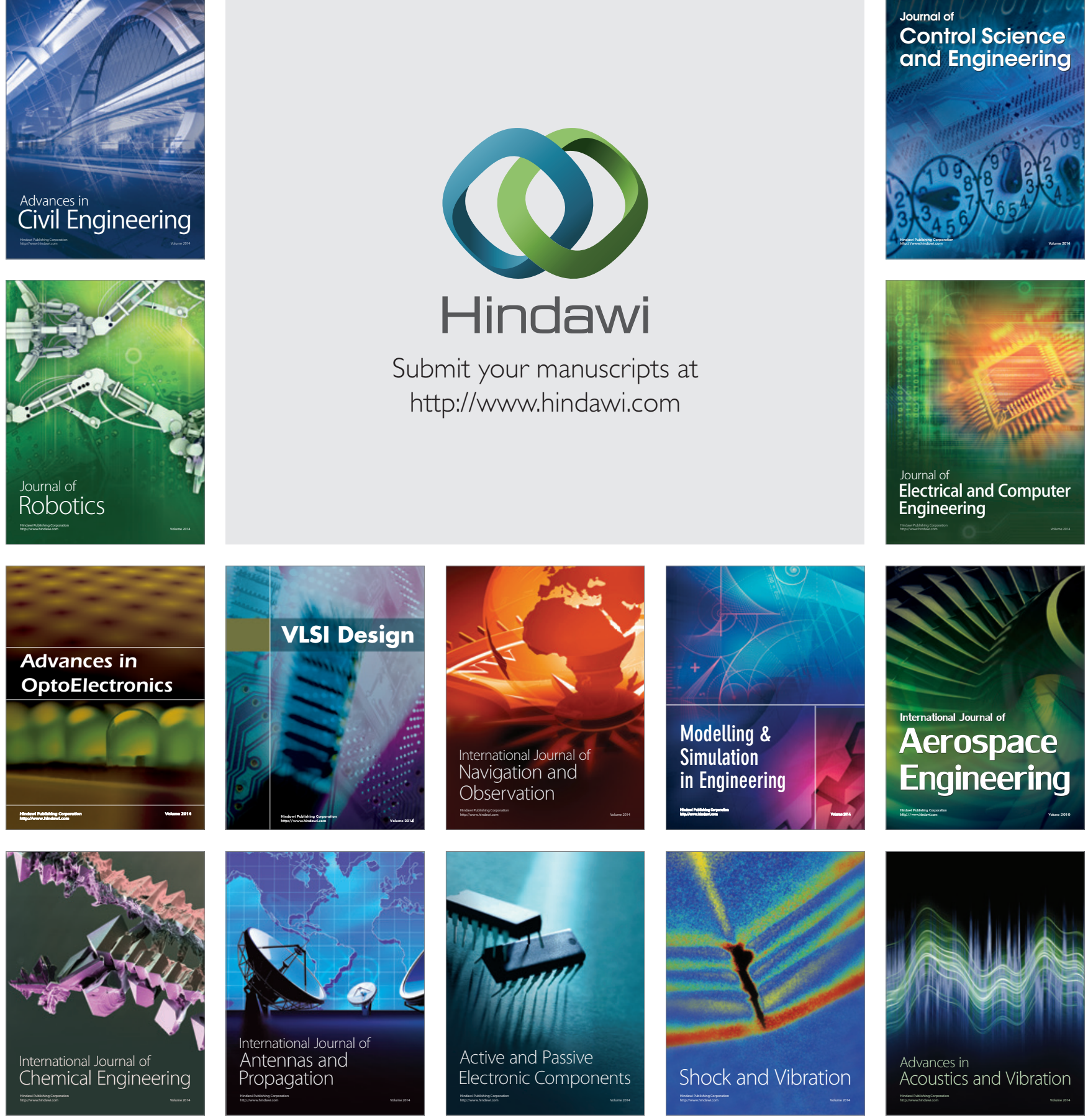